\title{
The emotion-induced memory trade-off: More than an effect of overt attention?
}

\author{
Katherine R. Mickley Steinmetz • Elizabeth A. Kensinger
}

Published online: 5 September 2012

(C) Psychonomic Society, Inc. 2012

\begin{abstract}
Although it has been suggested that many effects of emotion on memory are attributable to attention, in the present study we addressed the hypothesis that such effects may relate to a number of different factors during encoding or postencoding. One way to look at the effects of emotion on memory is by examining the emotion-induced memory trade-off, whereby enhanced memory for emotional items often comes at the cost of memory for surrounding background information. We present evidence that this trade-off cannot be explained solely by overt attention (measured via eyetracking) directed to the emotional items during encoding. Participants did not devote more overt attention to emotional than to neutral items when those items were selectively remembered (at the expense of their backgrounds). Only when participants were asked to answer true/false questions about the items and the backgrounds - a manipulation designed to affect both overt attention and poststimulus elaboration -was there a reduction in selective emotional item memory due to an increase in background memory. These results indicate that the allocation of overt visual attention during encoding is not sufficient to predict the occurrence of selective item memory for emotional items.
\end{abstract}

Keywords Memory $\cdot$ Attention $\cdot$ Emotion $\cdot$ Eye-tracking

Electronic supplementary material The online version of this article (doi:10.3758/s13421-012-0247-8) contains supplementary material, which is available to authorized users.

K. R. M. Steinmetz ( $\square)$

Department of Psychology, Wofford College,

429 North Church Street,

Spartanburg, South Carolina 29303, USA

e-mail: steinmetzkr@wofford.edu

E. A. Kensinger

Department of Psychology, Boston College,

McGuinn Hall 140 Commonwealth Avenue,

Chestnut Hill, MA 02467, USA
Emotional information is usually remembered better than neutral information (see Reisberg \& Heuer, 2004), but the mechanisms behind this enhancement are still unclear. The increased memory for emotional information may be due to a number of different factors. For example, emotional stimuli may be more likely to attract and sustain attention (Anderson, 2005; Calvo \& Lang, 2004), and emotional stimuli may also be more likely to evoke cognitive processing, elaboration, and rehearsal (Hamann, 2001; Libkuman, Stabler, \& Otani, 2004).

Many studies have provided evidence for the emotional prioritization of attention. People are quicker to notice emotional information within a visual array (Öhman, Flykt, \& Esteves, 2001), are more likely to notice emotional words that are presented very quickly (Anderson \& Phelps, 2001), and are more susceptible to interference from emotional than from neutral distractors (McKenna \& Sharma, 1995; Schimmack \& Derryberry, 2005). These behavioral effects of emotion on attention have also been shown in studies that have used eye gaze as a measure of overt visual attention: People tend to fixate first and to look longer at emotional pictures that are presented side by side with neutral pictures (Calvo \& Lang, 2004; Nummenmaa, Hyönä, \& Calvo, 2006). In addition, people fixate longer on irrelevant emotional distractor pictures than on neutral ones (Bannerman, Milders, \& Sahraie, 2009; Calvo \& Lang, 2004; Koster, Crombez, Verschuere, \& De Houwer, 2004; Nummenmaa et al., 2006; Sarter, Givens, \& Bruno, 2001). Thus, it seems that attention is initially focused on emotional information and that it is harder for participants to disengage attention from that information.

The attention mediation hypothesis of the emotional enhancement of memory (Talmi, Anderson, Riggs, Caplan, \& Moscovitch, 2008) and other attentional accounts (Cahill \& McGaugh, 1998; Hamann, 2001) suggest that emotional enhancements in memory are at least partly due to the increased attention directed toward emotional items at encoding. This theory has been supported by evidence that 
some brain regions, such as the fusiform gyrus, are conjointly activated for visual attention and memory enhancement for emotional information (Talmi et al., 2008). An attention-based theory has also been the dominant theory to explain the emotion-induced memory trade-off (see Reisberg \& Heuer, 2004, for a review), the finding that emotional items are often remembered at the expense of their contexts. The rationale behind this idea centers on the fact that emotional items that are high in arousal (i.e., exciting or stimulating) tend to capture attention and to get prioritized for processing (Christianson, 1992; Dolan \& Vuilleumier, 2003; Easterbrook, 1959; Schimmack \& Derryberry, 2005). Since more attentional resources are directed toward these higharousal emotional components, participants may be more likely to encode the emotionally arousing components of the scene and less likely to encode the other, neutral, contextual details. This focus may lead to selective memory for the emotional components (see Brown, 2003; Kensinger, Garoff-Eaton, \& Schacter, 2007a, b; Levine \& Edelstein, 2009; Safer, Christianson, Autry, \& Osterlund, 1998).

Although the emotion-induced memory trade-off is often discussed as being related to visual attention, previous research has not directly looked at the link between visual attention and selective item memory. Loftus, Loftus, and Messo (1987) did look at visual attention and memory separately, in reference to the "weapon focus" effect. In this study, participants' eye gaze was monitored while they were presented with slides in which a person entered a bank with either a gun or a check. Participants made more fixations on the gun than on the check when viewing the slides. In addition, memory for surrounding elements was poorer in the weapon condition than in the check condition. This is compelling evidence that attention at encoding may be allocated differently, depending on the presence of an emotional item. However, in this study the researchers looked at the eyegaze and memory results separately and did not directly relate eye gaze and memory. In other words, this study did not assess whether greater fixation on the weapon predicted better memory for the weapon and poorer memory for the surrounding elements in each trial. Thus, there was no indication that the increased fixation on the weapon (and the decreased fixation on the background) at encoding was what led to an increased likelihood of the weapon focus effect in memory. Therefore, it is unclear whether there is a causal link between attention allocation at encoding and the memory trade-off. In fact, a recent study suggested that attention allocation may not be the whole story: When a mediation analysis was used to examine the degree to which eye-gaze fixation upon an emotional item corresponded with later memory for that item, the analysis revealed that attention only partially mediated the enhancement in memory for negative items (Riggs, McQuiggan, Farb, Anderson, \& Ryan, 2011). Moreover, eye gaze on the emotional item did not relate to participants' poor ability to remember neutral items presented at the periphery of the emotional item. Thus, there is reason to believe that attention may not be the sole factor that causes the emotion-induced memory trade-off.

Given the recent evidence that attention only partially mediates these memory effects, one must consider other potential mediating factors. One such factor is poststimulus elaboration. The idea that poststimulus elaboration may drive the memory trade-off was first proposed by Christianson (1992), who suggested that the enhanced elaboration that goes on after the encoding of emotional items (as opposed to nonemotional items or backgrounds) might lead to an emotion-induced trade-off in memory for emotional items and peripheral contexts. Thus, it is possible that other factors besides attention may contribute to emotion's enhancement in memory.

The present study focused on instances in which participants remembered items but not the backgrounds that they were paired with, a pattern that we will refer to as selective item memory. This differs from the emotion-induced memory trade-off because "selective item memory" refers to all instances of when items are remembered at the exclusion of the background, regardless of whether the items are emotional or neutral. The first goal of the present study was to investigate the role of overt attention in selective item memory. We examined whether selective item memory was more likely to occur when eye gaze was directed toward the item rather than toward the background, and if so, whether this overt focus on the item was more closely tied to selective item memory for emotional items than for neutral ones.

Second, in this study we sought to determine whether manipulating stimulus evaluation and postencoding processing changes what is remembered about emotional scenes and how attention is directed toward emotional items. To investigate this possibility, participants were asked to answer true/false questions about both the item and the background after the picture had left the screen. We hypothesized that this manipulation would enhance encoding of all scene elements, including the backgrounds that had been paired with emotional scenes. We wanted to examine the extent to which the reduction in selective item memory for emotional scenes would be connected to changes in overt visual attention during scene viewing.

\section{Experiment 1}

Method

\section{Participants}

The data from 43 Boston College students (ages 18-21; 22 men, 21 women) were analyzed for this study. For four of 
the participants, no eyetracking data were collected due to eyetracker malfunction (although these participants completed the task with the eyetracker set up in the same way as did the others). Therefore, for the behavioral analyses all 43 participants were included, but for the eyetracking analyses only 39 participants were included (ages 18-21; 21 men, 18 women). The participants included in the analyses for this study were a subset of those tested as part of a larger study (Mickley Steinmetz, 2011) examining individual differences in anxiety levels. The participants included in the present analyses were those who had a Beck Depression Inventory (BDI-II; Beck, Ward, Mendelsohn, Mock, \& Erbaugh, 1961) score under 10 and a Beck Anxiety Inventory (BAI; Beck, Epstein, Brown, \& Steer, 1988) score under 19. These cutoffs were chosen according to normative data in order to exclude BDI-II scores at or above the 99th percentile (i.e., a cutoff score of 10; Knight, 2006) and BAI scores at or above the 90th percentile (i.e., a cutoff score of 19; Gillis, Haaga, \& Ford, 1995). The anxiety score cutoff was deliberately more conservative (i.e., excluded a greater proportion of participants) so as to make sure that the high-anxiety participants recruited as part of the larger sample were not included. All of the participants had normal or corrected-to-normal vision, were native English speakers, and had no history of a neuropsychological or psychiatric disorder. None listed that they were taking medications that affect the central nervous system.

\section{Materials}

The stimuli consisted of complex visual scenes that were created by placing images of positive, negative, and neutral items (people or objects) onto neutral background scenes (see Fig. 1). The stimulus set included items and backgrounds used in prior studies (Kensinger, Gutchess, \& Schacter, 2007a, b; Waring \& Kensinger, 2009; Waring, Payne, Schacter, \& Kensinger, 2010). Care was taken to make sure that the positive, negative, and neutral items were of comparable size and were balanced by approximate scene location. The neutrality of the backgrounds was confirmed via a pilot study using an independent group of raters/ participants. This study revealed that the average background ratings, grouped by the emotion category of the item that was paired with each background at study, were all considered neutral (average rating in the neutral range: rated between 5 and 5.5 on a scale from 1 to 10 , with 1 being extremely negative, 5 being neutral, and 10 being extremely positive). Each composite image was approximately $10 \times$ 13 in. and $700 \times 550$ pixels.

The items were 180 nameable, photographic-quality color images that were taken from photo clip art packages (Hemera Technologies, Quebec, Canada), from the
International Affective Picture System set (Lang, Bradley, $\&$ Cuthbert, 1997), and from other online databases of images. The items were broken down by valence according to ratings gathered in previous studies (and confirmed by ratings collected in a separate experiment). Valence was rated on a 9-point scale, with 9 being the most positive and 1 being the most negative. There were 60 positive items (mean valence $=6.02, S E=0.81$ ), 60 negative items (mean valence $=3.80, S E=0.82$ ), and 60 neutral items (mean valence $=5.29, S E=0.75$ ). Arousal was rated on a 5-point scale, with low numbers indicating soothing or subduing items and high numbers indicating exciting or agitating items (arousal mean $[S D]$ : positive $=3.02[0.57]$, negative $=3.19$ $[0.66]$, neutral $=2.35[0.61])$. Valence was rated on a broader scale than arousal because, while arousal is often divided into two categories (high and low), valence is often split into three categories (positive, negative, or neutral). The positive and negative items were matched on arousal and absolute valence (all $p \mathrm{~s}>.30$ ), and neutral items were considered less arousing than both positive and negative items (all $p \mathrm{~s}<.05$ ). Items were incorporated onto plausible backgrounds, so that the congruency between the item and the background was comparable for each emotion category of item. A norming study $(N=20)$ showed no significant differences in congruency across emotion categories (all $t \mathrm{~s}<1.7, p \mathrm{~s}>.05$ ). Across emotion categories, composite scenes also were matched for visual complexity and for the number of people, animals, and buildings included in the scene as assessed by two independent raters.

The composite scenes were divided across two different study sets with 90 scenes per list (30 with a negative item, 30 with a positive item, and 30 with a neutral item). To control for order effects, participants saw one of four counterbalanced orders. On each list, composite scenes were pseudorandomly organized such that no more than three scenes from the same emotion category appeared in a row. To create these lists, first the scenes were pseudorandomized twice, using a random number generator. Then two additional lists were created by taking the original two lists and reversing the order of each.

\section{Equipment and procedure}

The eyetracking apparatus was a SensoMotoric Instruments (SMI) eyetracker. Participants' left-eye-gaze patterns were tracked at $500 \mathrm{~Hz}$ by a SMI iView X Hi-Speed 1250 tracking column. Prior to the eyetracking session, participants filled out the consent form, a demographics questionnaire, an assessment of their state and trait anxiety, and an assessment of their depressive symptoms (i.e., the BAI, the BDI-II, and the State-Trait Anxiety Inventory [STAI; Spielberger, Gorsuch, Lushene, Vagg, \& Jacobs, 1983]). The participants then completed the tasks described below. 
Fig. 1 Samples of (top) the composite study stimuli (a negative, neutral, or positive item on a neutral background), (middle) the recognition test stimuli (old and new items and backgrounds presented separately), and (bottom) the area-of-interest selection for the eyetracking analyses

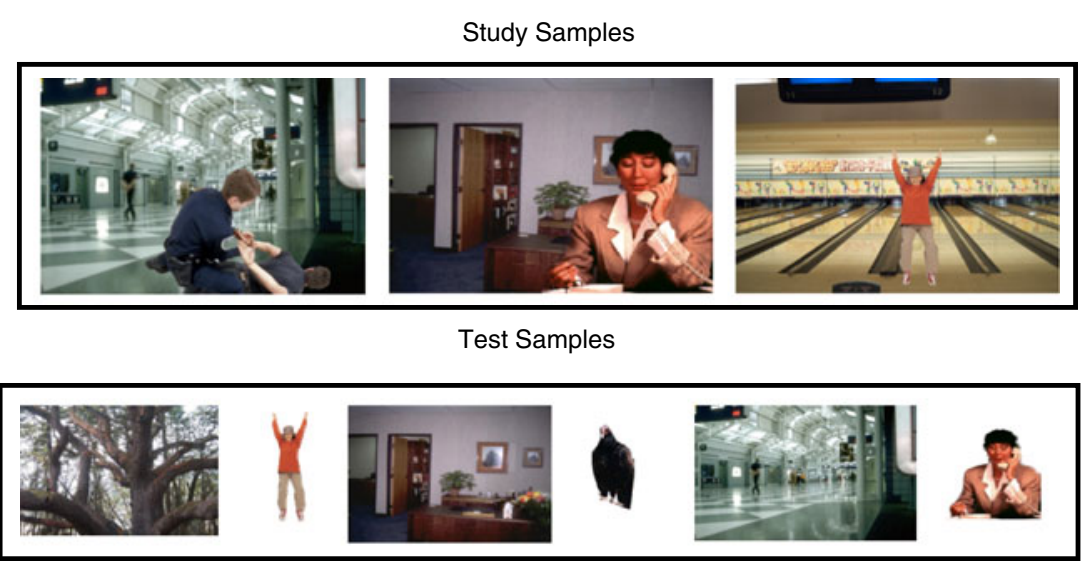

Area of Interest Selection Sample

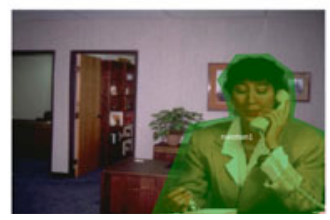

Encoding Task 1: View condition Participants were seated 2 feet away from a 14-in. computer screen at eye level with the center of the computer screen. Each participant's eye gaze was first tracked during a 17-point calibration. For the calibration, participants were asked to shift their gaze to 17 points on the computer screen to ensure that the eyetracker was accurately tracking the pupil of the left eye to all points on the computer screen. Once calibrated, the participants were given the task instructions. Participants were asked to look naturally at the screen as each picture appeared (as if they were watching television) and to look at the fixation cross in-between trials. During this incidental encoding session, each trial began with a white fixation cross presented on a black screen for $4,000 \mathrm{~ms}$. This was followed by the appearance of a composite picture for 5,000 ms. After a short practice (three pictures), the participants viewed 45 pictures (15 from each emotion category) following the randomization procedures described above.

Encoding Task 2: Poststimulus elaboration condition. Once the first set was completed, instructions were presented on the screen for the second incidental encoding task. Participants were instructed to look at each picture carefully while it was on the screen (for 5,000 ms) and were told that after the picture had been removed from the screen, two true/false questions would be presented one at a time. The participants were told that one true/false statement would be about the item in the scene (e.g., "The dog did not wear a collar") and that one would be about the background (e.g., "There were clouds in the sky"). Participants gave their "true" or "false" responses verbally so that they did not need to remove their gaze from the computer monitor. The experimenter recorded each response. Each participant then completed a short practice version of the encoding task and then went on to view the next 45 pictures (15 from each emotion category) using this incidental encoding task. The order of the encoding tasks was always constant, with the poststimulus elaboration condition administered after completion of the view condition, in order to avoid concerns that answering the true/false questions would alter the way in which participants viewed and elaborated upon the scenes in the view condition.

Delay and test phases. After participants had completed both phases of the encoding session, a battery of standardized cognitive tasks were administered, creating a retention delay of approximately $30 \mathrm{~min}$. The tests included the Shipley Vocabulary Test (Shipley, 1986), the Wechsler Adult Intelligence Scale Backward Digit Span Test (Wechsler, 1997), the Wechsler Adult Intelligence Scale Digit Symbol Test (Wechsler, 1997), the FAS Test of Phonemic Fluency (Spreen \& Benton, 1977), and the Stroop task (Stroop, 1935).

Following this delay, participants completed a surprise recognition test in which they viewed items and backgrounds extracted separately from the studied composite scenes, mixed with new items and backgrounds. The images included 90 studied items ( 30 of each valence), 90 studied backgrounds ( 30 studied with an item of each valence), 90 new items ( 30 of each valence), and 90 new backgrounds, for a total of 360 images (see Fig. 1).

Half of the studied items and backgrounds from each valence category had been presented in the view condition and half in the poststimulus elaboration condition. The 
Table 1 Experiment 1 memory data: Hits and false alarms (FAs) for each session

\begin{tabular}{|c|c|c|c|c|c|c|}
\hline \multirow{2}{*}{$\begin{array}{l}\text { Item } \\
\text { Valence }\end{array}$} & & & \multicolumn{2}{|c|}{ Session 1 (View) } & \multicolumn{2}{|c|}{ Session 2 (PSE) } \\
\hline & & & Item & Background & Item & Background \\
\hline \multirow[t]{4}{*}{ Positive } & Hits & Mean & .71 & .58 & .91 & .80 \\
\hline & & $S E$ & .03 & .03 & .02 & .02 \\
\hline & FAs & Mean & .08 & .12 & .08 & .12 \\
\hline & & $S E$ & .01 & .01 & .01 & .01 \\
\hline \multirow[t]{4}{*}{ Negative } & Hits & Mean & .73 & .58 & .93 & .82 \\
\hline & & $S E$ & .03 & .03 & .01 & .02 \\
\hline & FAs & Mean & .11 & .12 & .11 & .12 \\
\hline & & $S E$ & .02 & .01 & .02 & .01 \\
\hline \multirow[t]{4}{*}{ Neutral } & Hits & Mean & .61 & .63 & .85 & .79 \\
\hline & & $S E$ & .03 & .03 & .02 & .03 \\
\hline & FAs & Mean & .12 & .12 & .12 & .12 \\
\hline & & $S E$ & .01 & .01 & .01 & .01 \\
\hline
\end{tabular}

PSE, poststimulus elaboration

particular items and backgrounds that were old versus new were counterbalanced across participants according to the particular study lists that had been viewed. For each item or background, participants were asked to indicate whether they believed that the picture was new, whether they "remembered" it (recollected specific details of its presentation during the encoding session) or "knew" it (felt a sense of familiarity with the picture, without remembering details from the encoding session). This test was self-paced. For the behavioral results, all "remember" and "know" responses were collapsed together in order to get a measure of all items that the participant had cited as being "old."

Because it was not possible to fully counterbalance the categories of emotional items presented with each background (i.e., each background could not always be plausibly presented with a neutral, a negative, and a positive item), a pilot study $(N=14)$ was conducted to assess whether there was any difference in memorability for the backgrounds that we would later pair with each category of emotional item. In this pilot study, the backgrounds were presented alone (without an item) at study and test. The analyses were then conducted to look at memory for backgrounds based on what valence they were paired with. The results revealed no differences in memory for the backgrounds that would later be paired with positive, negative, or neutral items in the experiments (hit mean $[S D]$ : positive $=.82(.15)$, negative $=$ $.80(.13)$, neutral $=.83[.14]$; all $p \mathrm{~s}>.28)$.

\section{Data analysis}

For the behavioral memory data, false alarms (new pictures that were incorrectly cited as being old) were subtracted from hits (pictures that were correctly recognized as being old) in order to correct for any bias to call a picture "old." These corrected recognition scores were computed separately for each item type (positive, negative, or neutral) and for each background type (although note that there were different false alarm rates for positive, negative, and neutral items, but only one false alarm rate for backgrounds, because the valence of the backgrounds was defined by the valence of the item that it had been studied with; on their own, all backgrounds, including the new backgrounds, were neutral). Separate data for the hits versus the false alarms are presented in Table 1.

For the eyetracking results, the dependent variable of interest was the percentage of scene viewing time that participants fixated on the item, out of the total time spent fixating on the scene. The emotional or neutral items within the scene were defined as areas of interest (see Fig. 1, bottom). Fixation percentages were calculated by measuring the amount of time that a participant fixated on that predetermined area of interest and dividing that by the total amount of time that the participant fixated on any area of the scene. For example, if the participant spent $60 \%$ of the time fixating on the item and $20 \%$ of time fixating on the background, we would then divide the amount of time fixating on the item $(60 \%)$ by the total time fixating $(80 \%)$ to get a percent item fixation of $75 \%$. Please note that the item and background fixation times will never add

Table 2 Eyetracking percentages of item fixations for each experiment and session when selective item memory did and did not occur

\begin{tabular}{|c|c|c|c|c|c|c|c|c|c|c|c|c|c|}
\hline & & \multirow{2}{*}{\multicolumn{3}{|c|}{$\frac{\text { Experiment } 1}{\text { Session } 1 \text { (View) }}$}} & \multirow{2}{*}{\multicolumn{3}{|c|}{$\frac{\text { Experiment } 2}{\text { Session } 1 \text { (View) }}$}} & \multirow{2}{*}{\multicolumn{3}{|c|}{$\begin{array}{l}\text { Experiment } 2 \\
\text { Session } 2 \text { (View) }\end{array}$}} & \multicolumn{3}{|c|}{ Experiment 1} \\
\hline & & & & & & & & & & & \multicolumn{3}{|c|}{ Session 2 (Poststimulus Elaboration) } \\
\hline & & Pos & Neg & Neu & Pos & $\mathrm{Neg}$ & Neu & Pos & Neg & Neu & Pos & $\mathrm{Neg}$ & $\mathrm{Neu}$ \\
\hline \multirow[t]{2}{*}{ Selective item memory } & Mean & .66 & .58 & .63 & .70 & .69 & .72 & .66 & .63 & .69 & .55 & .44 & .47 \\
\hline & $S E$ & .03 & .03 & .03 & .02 & .02 & .02 & .02 & .01 & .02 & .04 & .04 & .05 \\
\hline \multirow[t]{2}{*}{ No selective item memory } & Mean & .55 & .53 & .48 & .56 & .54 & .52 & .54 & .55 & .53 & .52 & .45 & .49 \\
\hline & $S E$ & .03 & .03 & .03 & .03 & .03 & .03 & .03 & .03 & .04 & .03 & .03 & .04 \\
\hline
\end{tabular}


up to $100 \%$, due to saccades and blinks that cut into the fixation times and due to occasional fixations off of the screen. Fixations were defined as when the gaze was still for $50 \mathrm{~ms}$ or more. As recommended by eyetracking software manufacturers, the first fixation for each slide was excluded from the analysis, as the first fixation actually reflects the last fixation from the previous trial (i.e., the participant is usually still looking at where the fixation cross or text had been located on the previous screen). The BeGaze software was used to record the appearance and duration of the visual stimuli, as well as gazes and fixations to the stimuli.

In order to look directly at the link between eye gaze and selective item memory, item fixation at encoding was analyzed on the basis of later memory. These analyses compared instances in which selective item memory occurred versus instances in which it did not. To do this, percentages of item fixation times at encoding were computed separately for scenes that later showed selective item memory (e.g., the item was remembered and the background was forgotten) and for scenes that did not elicit selective item memory (e.g., the item was remembered as well as the background, or the item was forgotten and the background was remembered).

\section{Results}

\section{Behavioral results}

View condition. In order to analyze differences in memory for items and backgrounds as a function of emotion, a $3 \times 2$ ANOVA was conducted with the factors Emotion (positive, negative, or neutral) and Component Type (item or background; see Fig. 2a, black bars). This analysis revealed a main effect of component type $[F(1,42)=28.323, p<.001$, $\left.\eta_{P}^{2}=.403\right]$ and a marginal main effect of emotion $[F(2,84)=$ $\left.2.455, p<.095, \eta_{P}^{2}=.055\right]$, qualified by a Component Type $\times$ Emotion interaction $\left[F(2,84)=18.124, p<.001, \eta_{P}^{2}=.301\right]$. Subsequent $t$ tests revealed significantly better memory for the emotional items (collapsing across the positive and negative valence categories), as compared to the neutral items $[t(42)=5.812, p<.001]$. For the backgrounds, the opposite was true: We found worse memory for backgrounds previously paired with positive or negative items (collapsed) than for backgrounds previously paired with neutral items $[t(42)=$ $2.171, p<.05]$. Thus, an emotion-induced memory trade-off existed: There was an increase in memory for emotional items ${ }^{1}$ as compared to neutral ones. In addition, we found a decrease in memory for backgrounds that had previously been

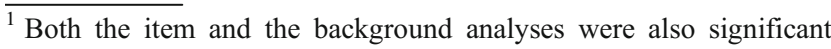
when positive and negative items were compared individually to neutral items.
}

paired with emotional items, as compared to those that had been paired with neutral items. There was no significant difference in memory between positive and negative items or between their accompanying backgrounds (all $t \mathrm{~s}<0.5, p \mathrm{~s}>.5)$.

Comparing the view to the poststimulus elaboration condition. In order to examine the effect of the poststimulus elaboration condition (i.e., when participants answered the true/false questions about the item and the background), a $3 \times 2 \times 2$ ANOVA was conducted with the factors Emotion (positive, negative, or neutral), Poststimulus Elaboration Condition (view or poststimulus elaboration), and Component Type (item or background; see Fig. 2b, black bars). This analysis revealed a main effect of emotion $[F(2,84)=8.235$, $\left.p<.001, \eta_{P}^{2}=.164\right]$, a main effect of poststimulus elaboration $\left[F(1,42)=201.398, p<.001, \eta_{P}^{2}=.827\right]$, and a main effect of component type $\left[F(1,42)=50.51, p<.001, \eta_{P}^{2}=.546\right]$. These main effects were qualified by an Emotion $\times$ Component Type interaction $\left[F(2,84)=15.771, p<.001, \eta_{P}^{2}=.273\right]$ and by a three-way Emotion $\times$ Poststimulus Elaboration $\times$ Component Type interaction $[F(2,84)=4.798, p<.05$, $\left.\eta_{P}^{2}=.103\right]$.

This three-way interaction arose because under the view condition there was an emotion-induced memory trade-off (increased emotional item memory, decreased memory for backgrounds), whereas under the poststimulus elaboration condition this was no longer the case. Separate ANOVAs conducted for items and backgrounds confirmed that there was always a main effect of emotion on item memory $\left[F(2,84)=19.686, p<.001, \eta_{P}^{2}=.319\right]$, such that there was better memory for positive $[t(42)=5.024, p<.001]$ and negative $[t(42)=6.045, p<.001]$ items than for neutral items in both conditions. There was no significant interaction between emotion and poststimulus elaboration $(F<1.5$, $p>$.2). However, for backgrounds, there was a marginal Poststimulus Elaboration $\times$ Emotion interaction $[F(2,84)=$ $\left.2.685, p=.074, \eta_{P}^{2}=.06\right]^{2}$ : While in the view condition, memory was significantly worse for backgrounds previously paired with emotional items than for those paired with neutral items $[t(42)=2.171, p<.05]$, in the poststimulus elaboration condition there was no significant difference between these two types of backgrounds $(t<1.0, p>.3)$.

\footnotetext{
${ }^{2}$ An analysis was also conducted in which only the individuals who were the most likely to show the emotion-induced memory trade-off were included. This was determined by taking a median split of a composite memory trade-off score. This interaction did reach significance when only individuals who exhibited a high trade-off under the view condition were considered in the analysis $[F(2,38)=11.947$, $\left.p<.0001, \eta_{P}^{2}=.39\right]$. Full "high-trade-off" analyses are presented in the supplementary data.
} 
Fig. 2 Behavioral memory data (hits - false alarms) for (a) the first session (view condition [Exps. 1 and 2]) and (b) the second session (poststimulus elaboration [Exp. 1] and view condition [Exp. 2])

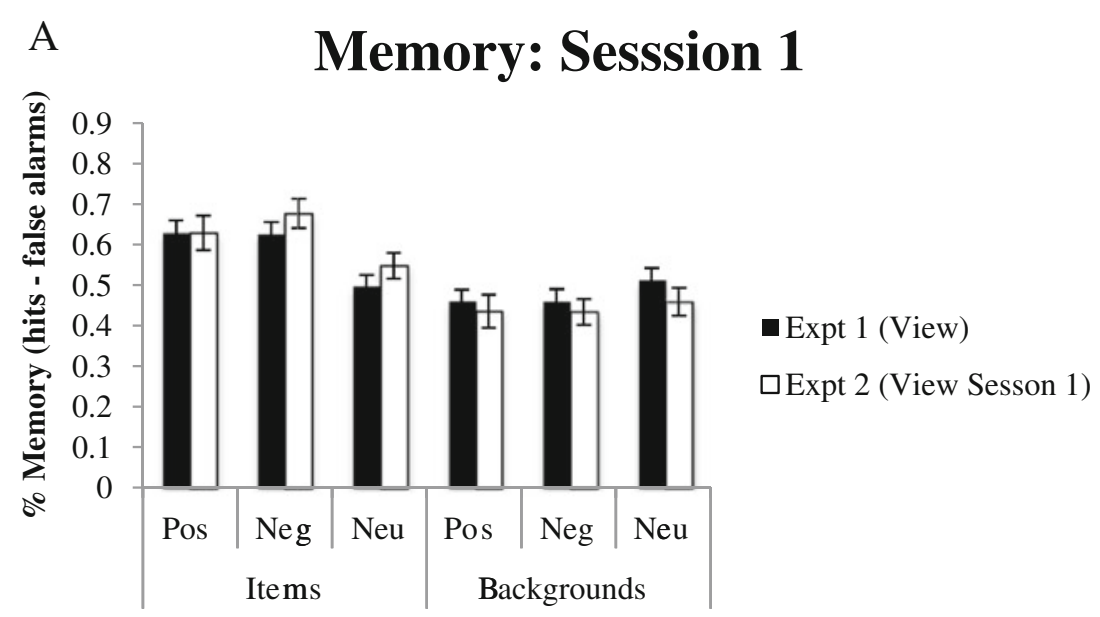

B Memory: Sesssion 2

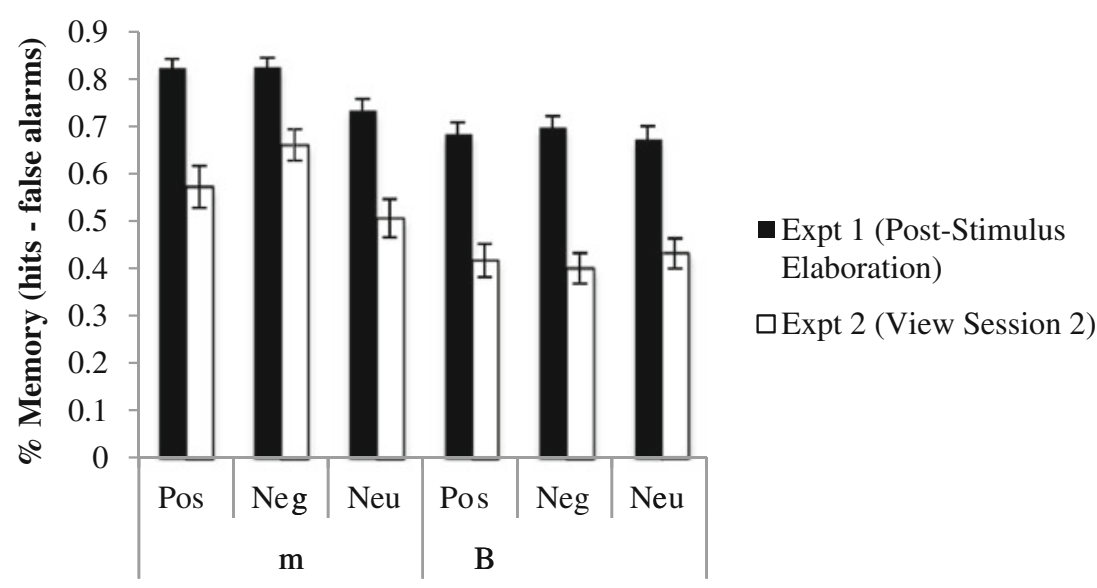

Eye-gaze results

View condition In order to determine the relationship between the percentage of time spent fixating on the item and the likelihood of remembering the item but not the background (i.e., selective item memory), we analyzed the item fixation data using a Selective Item Memory (present or absent) $\times$ Emotion (positive, negative, or neutral) ANOVA. The eye-gaze data from 36 participants were included in this analysis due to missing data (e.g., three participants never exhibited selective item memory for scenes of a particular emotion). This analysis revealed a main effect of selective item memory $\left[F(1,35)=56.271, p .001, \eta_{P}^{2}=.617\right]$ and a main effect of emotion $\left[F(2,70)=4.028, p .05, \eta_{P}^{2}=.103\right]$, but not an Emotion $\times$ Selective Item Memory interaction ${ }^{3}$

\footnotetext{
${ }^{3}$ We still found no Emotion $\times$ Selective Item Memory interaction when all old items were restricted to just those trials on which participants gave a "remember" response, indicating that they had a detailed recollection of the stimulus. Only 18 participants could be included in this analysis, due to missing data; some of the participants had no "remember" responses for particular conditions.
}

$(F<1.9, p>.15$; see Table 2 and Fig. 3). Subsequent $t$ tests revealed that percentages of fixations on the item were greater in scenes for which selective item memory later occurred, as compared to those for which there was no selective item memory $[t(35)=6.149, p<.001]$. The effect of emotion was such that people fixated longer on positive than on negative items $[t(35)=2.112, p<.05]$ and marginally longer on positive than on neutral items $[t(35)=1.718, p<.1]$. Although we found no Emotion $\times$ Selective Item Memory interaction, the results suggest that the effect of emotion was, if anything, more pronounced when there was not selective item memory, with the difference between positive- and neutral-item fixation times differing only in that condition $[t(35)=2.692, p<.05]$ and not in the selective item memory condition $(t<1, p>.1)$.

Poststimulus elaboration condition An Emotion (positive, negative, or neutral) $\times$ Poststimulus Elaboration Condition (view or poststimulus elaboration) $\times$ Selective Item Memory (present or absent) ANOVA was conducted. Only a subset of participants $(N=21)$ could be included in this analysis, due 


\section{Experiment 1 - Session 1 (View)}

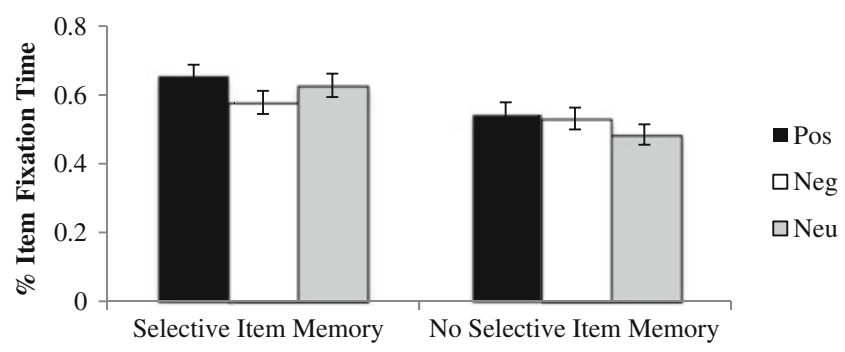

Experiment 2 - Session 1 (View)

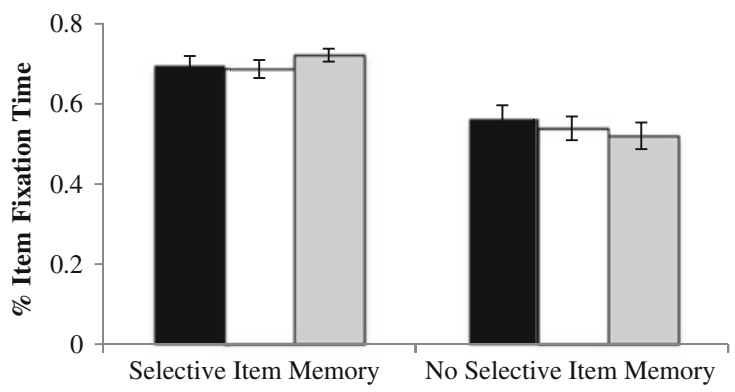

Experiment 2 - Session 2 (View)

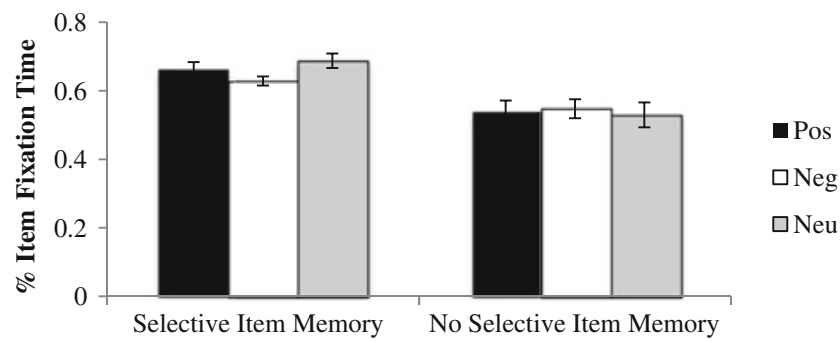

Fig. 3 Percentages of time spent fixating on the item when the item was positive, negative, or neutral and when selective item memory (memory for the item but not the background) occurred or did not occur

to missing data (i.e., some people never exhibited selective item memory for neutral scenes under the poststimulus elaboration condition). This analysis revealed a main effect of selective item memory $[F(1,20)=36.316, p<.001$, $\left.\eta_{P}^{2}=.645\right]$, a main effect of poststimulus elaboration condition $\left[F(1,20)=5.354, p .05, \eta_{P}^{2}=.211\right]$, and a main effect of emotion $\left[F(2,40)=7.862, p .01, \eta_{P}^{2}=.282\right.$; see Table 2]. These effects were such that we found longer fixation times when selective item memory occurred than when it did not $[t(38)=7.110, p<.001]$, longer item fixation times under the view than under the poststimulus elaboration condition $[t(38)=3.135, p<.01]$, longer fixations on positive and neutral than on negative items [positive, $t(38)=4.951$, $p<.05$; neutral, $t(38)=1.795, p<.05$ ], and marginally longer fixations on positive than on neutral items $[t(38)=$ $1.795, p<.09]$. There were no interactions among the factors.

\section{Discussion}

The first goal of this experiment was to determine whether overt attention explains emotion enhancements in selective item memory. When participants were asked to view pictures naturally at encoding, selective item memory occurred more often for scenes that included emotional items than for those containing neutral items (i.e., the emotion-induced memory trade-off). However, overt visual attention did not predict this memory enhancement: There were not increased fixations on the emotional vs. neutral items for which participants exhibited selective item memory, as would have been expected if visual attention were the sole driver of the memory trade-off.

The second goal of this experiment was to determine whether a manipulation that changes the strength of the memory trade-off does so by changing the overt attention directed toward emotional items. In the poststimulus elaboration condition, participants answered true/false questions about both the item and the background in the poststimulus period. Under this manipulation, memory for both the items and the backgrounds was improved. Memory was especially improved for the backgrounds that had been paired with emotional items, and thus the memory trade-off was eliminated, because there was no longer poorer memory for the backgrounds that had been presented with an emotional item. Importantly, this specific change in memory for the backgrounds of emotional scenes was not mirrored by a change in overt attention for the emotional scenes. Overall, participants looked for shorter periods at items during the poststimulus elaboration condition than during the view condition, but this effect of condition did not interact with the emotional value of the item.

These results suggest that eye gaze does not explain selective memory for emotional items; overt attention is no more likely to be directed toward emotional items when they are selectively remembered than when they are not. Moreover, the effect of emotion on overt attention did not differ in conditions under which selective item memory occurred more often (the view condition) and less often (the poststimulus elaboration condition).

Because of this novel result of no increased fixation times for emotional items when selective item memory occurred, we sought to replicate this finding in a second experiment that would include more trials within the view condition. Increasing the number of trials in the view condition should provide more measurement accuracy to examine whether emotion influences the relation between eye gaze and selective item memory. The second experiment could also clarify another potentially problematic design element of Experiment 1 , which was that the poststimulus elaboration condition always came second, so that the instructions in that condition would not influence how participants attended to the scenes in the view condition. Since the poststimulus elaboration 
condition always came second, it is possible that the differences in memory between the view and poststimulus elaboration conditions could be due to fatigue or habituation. In Experiment 2, we could compare the view trials from the first and second halves of the experiment, providing an opportunity not only to replicate the findings of Experiment 1, but also to rule out order effects.

\section{Experiment 2}

\section{Method}

The data from 37 Boston College students (ages 18-21; 12 men, 25 women) were analyzed for this study. The methods for Experiment 2 were identical to those from Experiment 1, except that instead of having the view condition followed by the poststimulus elaboration condition, participants viewed the pictures for both halves of the encoding session.

\section{Results}

\section{Behavioral results}

Separate data for the hits and false alarms in this experiment may be found in Table 3. To determine whether there were differences in memory between the first and second halves of the encoding session, a $3 \times 2 \times 2$ ANOVA was conducted with the factors Emotion (positive, negative, or neutral), Component Type (item or background), and View Session (first or second half). This analysis revealed a main effect of component type $\left[F(1,36)=94.376, p<.0001, \eta_{P}^{2}=.724\right]$ and a main effect of emotion $[F(2,72)=7.449, p<.01$,

Table 3 Experiment 2 memory data: Hits and false alarms (FAs) for each session

\begin{tabular}{|c|c|c|c|c|c|c|}
\hline \multirow{2}{*}{$\begin{array}{l}\text { Item } \\
\text { Valence }\end{array}$} & & & \multicolumn{2}{|c|}{ Session 1 (View) } & \multicolumn{2}{|c|}{ Session 2 (View) } \\
\hline & & & Item & Background & Item & Background \\
\hline \multirow[t]{4}{*}{ Positive } & Hits & Mean & .73 & .56 & .68 & .53 \\
\hline & & $S E$ & .03 & .04 & .03 & .03 \\
\hline & FAs & Mean & .10 & .11 & .10 & .11 \\
\hline & & $S E$ & .02 & .02 & .02 & .02 \\
\hline \multirow[t]{4}{*}{ Negative } & Hits & Mean & .76 & .55 & .75 & .52 \\
\hline & & $S E$ & .03 & .03 & .03 & .03 \\
\hline & FAs & Mean & .08 & .11 & .08 & .11 \\
\hline & & $S E$ & .02 & .02 & .02 & .02 \\
\hline \multirow[t]{4}{*}{ Neutral } & Hits & Mean & .63 & .58 & .59 & .55 \\
\hline & & $S E$ & .03 & .03 & .04 & .03 \\
\hline & FAs & Mean & .08 & .11 & .08 & .11 \\
\hline & & $S E$ & .02 & .02 & .02 & .02 \\
\hline
\end{tabular}

$\left.\eta_{P}^{2}=.171\right]$, qualified by the typical Component Type $\times$ Emotion interaction seen in this paradigm $[F(1,36)=20.889$, $\left.p<.001, \eta_{P}^{2}=.367\right]$, indicative of memory enhancement for emotional items but not for their corresponding backgrounds (see Fig. 2, white bars). The ANOVA also revealed a main effect of view session $\left[F(1,36)=4.804, p<.05, \eta_{P}^{2}=.118\right]$, with better memory for picture components viewed in the first as compared to the second half of the session. Critically, we found no interactions with the view session $(F<0.4, p>.6)$.

Comparing across experiments. Although the lack of any interaction with view session suggests that the dissipation of the trade-off in the poststimulus elaboration condition was not simply due to time-on-task effects, a more direct test of this hypothesis would be to directly compare Experiment 2 (E2) to Experiment 1 (E1). Thus, an Emotion (positive, negative, or neutral $) \times$ Experiment $(E 1$ or E2) $\times$ Session (Part 1 or 2$) \times$ Component Type (item or background) ANOVA was conducted (see Fig. 2). All of the main effects were significant $\left(F_{\mathrm{s}}>12, p \mathrm{~s}<.01\right)$. These main effects were qualified by two two-way interactions. First, there was a Session $\times$ Experiment interaction $[F(1,77)=136.04, p<.001$, $\left.\eta_{P}^{2}=.639\right]$, such that only for the second session (poststimulus elaboration in E1, view in E2) was there better memory in $\mathrm{E} 1$ than in $\mathrm{E} 2$ [second session; $t(77)=65.971, p<.001$ ] Second, we found an interaction between experiment and component type $\left[F(1,77)=6.780, p<.05, \eta_{P}^{2}=.081\right]$, with the poststimulus elaboration condition enhancing memory for backgrounds more than it enhanced memory for items. These two-way interactions were qualified by a marginal three-way interaction among experiment, component type, and emotion $\left[F(2,154)=2.472, p=.08, \eta_{P}^{2}=.031\right]$ and a marginal four-way interaction $[F(2,154)=2.418, p=.092$, $\left.\eta_{P}^{2}=.03\right] .{ }^{4}$ These interactions were such that for the second session (poststimulus elaboration in E1, view in E2), the emotional backgrounds produced better memory in E1 than in E2.

\section{Eye-gaze results}

In order to determine the relationship between percentages of item fixations and selective item memory and to determine whether fixation differences occurred between the first and second halves of the encoding session, a Selective Item Memory (present or absent) $\times$ Emotion (positive, negative, or neutral) $\times$ View Session (first or second half) ANOVA was conducted. This analysis included data from 29

\footnotetext{
${ }^{4}$ This four-way interaction between experiment, session, component type, and emotion reached significance only the individuals above the median split for the composite memory trade-off scores were computed $\left(F(2,70)=6.808, p<.002, \eta_{P}^{2}=.163\right)$.
} 
individuals, due to missing data for some participants in some conditions. The ANOVA revealed a main effect of selective item memory $\left[F(1,28)=73.741, p<.0001, \eta_{P}^{2}=.725\right]$ and a marginal main effect of emotion $[F(2,56)=2.965$, $\left.p=.06, \eta_{P}^{2}=.096\right]$, qualified by a Selective Item Memory $\times$ Emotion interaction $^{5}\left[F(2,56)=3.284, p<.05, \eta_{P}^{2}=.105\right.$; see Fig. 3 and Table 2]. This interaction was such that, for neutral items, there was a $40 \%$ increase in item fixation times when selective item memory occurred, as compared to when there was not selective item memory. However, for the positive and negative items, there were only $24.5 \%$ and $23.9 \%$ increases, respectively. Thus, consistent with Experiment 1, overt attention did not relate to selective emotional item memory, and in fact attention was more closely related to selective neutral item memory. There were no differences in fixation percentages between the first and second sessions, in the form of either main effects or interactions (all $F_{\mathrm{s}}<1.5, p \mathrm{~s}>.3$ ).

Comparison across experiments In order to compare the two experiments directly, an Emotion (positive, negative, or neutral) $\times$ Selective Item Memory (present or absent) $\times$ Experiment $(\mathrm{E} 1$ or E2) $\times$ Session $($ Part 1 or 2$)$ ANOVA was conducted for item fixation percentages. This ANOVA revealed a main effect of emotion $[F(2,148)=4.845, p<.01$, $\left.\eta_{P}^{2}=.061\right]$, such that participants looked longer at positive $[t(75)=2.843, p<.01]$ or neutral $[t(75)=1.944, p<.06]$ items than at negative items when selective item memory occurred. It also revealed main effects of selective item memory $\left[F(1,74)=58.908, p<.001, \eta_{P}^{2}=.443\right]$, session $\left[F(1,74)=14.797, p<.001, \eta_{P}^{2}=.167\right]$, and experiment $\left[F(1,74)=12.195, p<.001, \eta_{P}^{2}=.141\right]$. These main effects were qualified by a Selective Item Memory $\times$ Experiment interaction $\left[F(1,74)=12.737, p<.001, \eta_{P}^{2}=.443\right]$, such that looking times were longer for Experiment 1 than for Experiment 2 only when there was selective item memory $[t(74)=4.437, p<.001]$. We also found a Session $\times$ Experiment interaction $\left[F(1,74)=5.243, p<.05, \eta_{P}^{2}=.066\right]$, such that participants looked longer at items during the second session under the view instructions than under the poststimulus elaboration instructions $[t(74)=4.528, p<.001$; see Table 2].

\section{Discussion}

The goal of Experiment 2 was to replicate the finding that overt visual attention did not explain selective item memory for emotional items and to ensure that the condition effects

\footnotetext{
$\overline{{ }^{5} \text { This Emotion }} \times$ Selective Item Memory interaction was not present when all old items were restricted to just those trials on which participants gave a "remember" response, indicating that they had a detailed recollection of the stimulus. Only 13 participants were included in this analysis, due to the remember-only restriction.
}

revealed in Experiment 1 did not simply reflect order effects. This had been a concern because the poststimulus elaboration phase in Experiment 1 had always come second so that it wouldn't bias processing in the view condition. The results from Experiment 2 revealed that the session (Part 1 vs. 2) did not affect either the memory or eye-gaze patterns. This indicates that habituation, practice, or timeon-task effects most likely do not explain the effects of poststimulus elaboration in Experiment 1.

Importantly, similar eye-gaze results were found in both Experiments 1 and 2: Participants looked longer at items for which they exhibited selective item memory. However, just as in Experiment 1, there was no specific enhancement in eye gaze for emotional items that exhibited selective item memory. This pattern of results remained true when both experiments were combined and when only people who were more likely to exhibit a memory trade-off were examined (see the supplementary data for the latter analyses). These findings indicate that there is no strong relation between the effects of emotion on selective item memory and the effects of emotion on eye gaze.

\section{General discussion}

In the present study, we examined the contribution of attention to selective item memory, examining why emotional items are more likely than neutral items to be remembered without their surrounding contextual details. The results of this study emphasized that although attention allocation is different when participants remember items without their backgrounds than when they remember both scene components, this difference is actually greater for neutral than for emotional items. Thus, changes in eye gaze do not appear to explain why selective item memory is more likely to occur for emotional items. Furthermore, even a manipulation that modified the emotion-induced memory trade-off did not disproportionately change overt attention for emotional items. This provides further evidence that the emotioninduced memory trade-off is not solely related to changes in visual attention. We suggest that a more complex combination of factors may lead individuals to be more likely to remember emotional items and not their contexts.

Attention allocation and selective item memory

The eye-gaze results revealed that selective item memory was associated with increased item fixation times: Participants looked longer at items when they remembered only those items and not their backgrounds. This finding is consistent with studies that have indicated that increased fixation times on a certain part of a complex scene may lead to better memory for that particular portion of the scene (Krugman, 
Fox, Fletcher, Fischer, \& Rojas, 1994). Thus, information that gets privileged attention at encoding is what tends to be remembered later.

However, in the present study we found that this relation was not stronger for emotional than for neutral items and that, if anything, the opposite was true. These results counter an attention-based model of the trade-off effect, which predicts that the relation between selective item memory and enhanced fixation times would be particularly strong for emotional items. The present findings can be reconciled with those of the Loftus et al. (1987) study, which revealed that when the eye-gaze and memory data were considered separately, participants both looked longer at an emotional object (e.g., a weapon) than at a neutral object (e.g., a check), and also were more likely to remember weapons at the expense of the surrounding information. The present study found similar results when eye-gaze data and memory data were considered separately: Across all scenes, participants looked longer at emotional items than at neutral items, and participants also showed more instances of selective item memory for emotional than for neutral items. However, in the present study, when the eye-gaze data were sorted by the occurrence of selective item memory, there was no evidence that the occurrence of selective item memory was more strongly tied to overt attention toward emotional items than neutral items. Thus, overt visual attention (eye gaze) is unlikely to explain why selective item memory occurs more often for emotional than for neutral items.

We are not the first to propose that attention is not the only factor influencing emotional memory. For example, Christianson, Loftus, Hoffman, and Loftus (1991) tracked participants' gazes while they viewed a slide show with a critical emotional slide. Christianson et al. found that even when eye gaze was limited to only one fixation for the critical emotional slide, participants still had better memory for the emotional slide than for the neutral one, suggesting that other factors may enhance emotional memory when attention is restricted. Furthermore, Talmi et al. (2008) found that even when they controlled for the amounts of attention given to negative and neutral pictures, participants still remembered negative pictures better. Most recently, Riggs et al. (2011) conducted a study that looked at the effect of attention on memory for central versus peripheral details. In this study, participants' eyes were tracked as they viewed emotional and neutral scenes, which were surrounded by three discrete and unrelated neutral objects. When participants' memory for the scenes and the objects was tested, Riggs et al. found selective item memory and increased fixations on emotional items. However, a mediation analysis revealed that attention only partially mediated emotion-enhanced memory for central pictures and did not explain participants' poor memory for the neutral objects in the periphery. Thus, in this previous study, which used a very different paradigm to study selective item memory, the researchers also found that enhanced memory for an emotional element may not be solely due to changes in attention at encoding.

\section{Poststimulus elaboration and selective item memory}

We hypothesized that the poststimulus elaboration condition in Experiment 1 would modify the emotion-induced memory trade-off. This manipulation, in which participants were asked true/false questions in the postencoding period, most likely modified both attentional and postencoding processes. As participants knew that they would be quizzed on both the items and backgrounds, they were more likely to look around the entire scene. In addition, answering questions about both the item and the background forced participants to continue to elaborate on both elements of the scene. As predicted, this manipulation improved memory for all scene elements, including a strong benefit for the backgrounds of emotional scenes. Critically, this behavioral change in memory was not accompanied by a similar change in the eyegaze data: That is, the change in memory was not related to a disproportionate change in attentional focus on the emotional items. This provides further evidence that factors aside from attention are related to selective item memory for emotional information.

One of these additional processes may be emotion's influence on poststimulus elaboration. Emotion tends to be associated with increased elaboration, and this may lead to an enhancement in memory for the emotional item, while the unelaborated context is forgotten. Although this is a plausible explanation, there is no consensus as to the role of poststimulus elaboration on emotional memory. While some studies have shown that limiting poststimulus elaboration may decrease emotional memory enhancement (Hulse, Allan, Memon, \& Read, 2007; Libkuman et al., 2004), others have shown no influence (Harris \& Pashler, 2005). While these studies focused on the effects of poststimulus elaboration on memory for the emotional item, there is a dearth of data assessing the effect of poststimulus elaboration on the contextual details of emotional events. Thus, more research is still needed in order to understand the full extent of influences such as poststimulus elaboration on the emotion-induced memory trade-off.

\section{Conclusion}

The present study indicated that, contrary to past assumptions, selective item memory for emotional information is not strongly related to attention at encoding. Although participants did devote more overt attention to emotional than to neutral items, this was equally true regardless of whether 
both items and backgrounds were remembered or whether items were remembered without the backgrounds that they had previously been paired with. Moreover, the effect of emotion on overt attention did not differ in conditions in which selective item memory occurred more often (the view condition) or less often (the poststimulus elaboration condition). These data suggest that, instead of a simple attentionbased mechanism, the mechanism behind selective item memory for emotional information must be more complex, perhaps including the way that information is elaborated.

Author note This research was supported by Grant No. BCS 0963581 from the National Science Foundation (to E.A.K.) and by a Psi Chi Graduate Research Grant (to K.R.M.S.). We thank Carolyn Humber, Joana Bueno, Christopher Stare, Sondra Corgan, and Michael O'Hara for their assistance with participant recruitment, testing, and data management. We also thank David Smith, Scott Slotnick, Maya Tamir and Jessica Payne for helpful discussion. Portions of the manuscript were included in a dissertation submitted by K.R.M.S.

\section{References}

Anderson, A. K. (2005). Affective influences on the attentional dynamics supporting awareness. Journal of Experimental Psychology: General, 134, 258-281. doi:10.1037/0096-3445.134.2.258

Anderson, A. K., \& Phelps, E. A. (2001). Lesions of the human amygdala impair enhanced perception of emotionally salient events. Nature, 411, 305-309.

Bannerman, R. L., Milders, M., \& Sahraie, A. (2009). Processing emotional stimuli: Comparison of saccadic and manual choicereaction times. Cognition \& Emotion, 23, 930-954. doi:10.1080/ 02699930802243303

Beck, A. T., Epstein, N., Brown, G., \& Steer, R. A. (1988). An inventory for measuring clinical anxiety: Psychometric properties. Journal of Consulting and Clinical Psychology, 56, 893-897.

Beck, A. T., Ward, C. H., Mendelsohn, M., Mock, J., \& Erbaugh, J. (1961). An inventory for measuring depression. Archives of General Psychology, 4, 561-571.

Brown, J. M. (2003). Eyewitness memory for arousing events: Putting things into context. Applied Cognitive Psychology, 17, 93-106.

Cahill, L., \& McGaugh, J. L. (1998). Mechanisms of emotional arousal and lasting declarative memory. Trends in Neurosciences, 21, 273-313.

Calvo, M. G., \& Lang, P. J. (2004). Gaze patterns when looking at emotional pictures: Motivationally biased attention. Motivation and Emotion, 28, 221-243.

Christianson, S.-Å. (1992). Emotional stress and eyewitness testimony: A critical review. Psychological Bulletin, 112, 284-309. doi:10.1037/0033-2909.112.2.284

Christianson, S.-Å., Loftus, E. F., Hoffman, H., \& Loftus, G. R. (1991). Eye fixations and memory for emotional events. Journal of Experimental Psychology: Learning, Memory, and Cognition, 17, 693-701. doi:10.1037/0278-7393.17.4.693

Dolan, R. J., \& Vuilleumier, P. (2003). Amygdala automaticity in emotional processing. Annals of the New York Academy of Sciences, 985, 348-355.

Easterbrook, J. A. (1959). The effects of emotion on cue utilization and the organization of behavior. Psychological Review, 66, 183-201. doi:10.1037/h0047707
Gillis, M. M., Haaga, D. A. F., \& Ford, G. T. (1995). Normative values for the Beck Anxiety Inventory. Fear Questionnaire, Penn Sate Worry Questionnaire, and Social Phobia and Anxiety inventory, Psychological Assessment, 4, 450-455.

Hamann, S. (2001). Cognitive and neural mechanisms of emotional memory. Trends in Cognitive Sciences, 5, 394-400.

Harris, C. R., \& Pashler, H. (2005). Enhanced memory for negatively emotionally charged pictures without selective rumination. Emotion, 5, 191-199. doi:10.1037/1528-3542.5.2.191

Hulse, L. M., Allan, K., Memon, A., \& Read, J. D. (2007). Emotional arousal and memory: A test of the poststimulus processing hypothesis. The American Journal of Psychology, 120, 73-90.

Kensinger, E. A., Garoff-Eaton, R. J., \& Schacter, D. L. (2007a). Effects of emotion on memory specificity: Memory trade-offs elicited by negative visually arousing stimuli. Journal of Memory and Language, 56, 575-591. doi:10.1016/j.jml. 2006.05.004

Kensinger, E. A., Gutchess, A. H., \& Schacter, D. L. (2007b). Effects of aging and encoding instructions on emotion-induced memory trade-offs. Psychology and Aging, 22, 781-795. doi:10.1037/ 0882-7974.22.4.781

Knight, R. G. (2006). Some general population norms for the short form Beck Depression Inventory. Journal of Clinical Psychology, 40, 751-753.

Koster, E. H. W., Crombez, G., Verschuere, B., \& De Houwer, J. (2004). Selective attention to threat in the dot probe paradigm: Differentiating vigilance and difficulty to disengage. Behaviour Research and Therapy, 42, 1183-1192. doi:10.1016/ j.brat.2003.08.001

Krugman, D. M., Fox, R. J., Fletcher, J. E., Fischer, P. M., \& Rojas, T. H. (1994). Do adolescents attend to warnings in cigarette advertising? An eye-tracking approach. Journal of Advertising Research, 34, 39-52.

Lang, P. J., Bradley, M. M., \& Cuthbert, B. N. (1997). International Affective Picture System (IAPS): Technical manual and affective ratings (Technical Report No. A-1). Gainesville: University of Florida, Center for Research in Psychophysiology.

Levine, L. J., \& Edelstein, R. S. (2009). Emotion and memory narrowing: A review and goal-relevance approach. Cognition \& Emotion, 23, 833-875. doi:10.1080/02699930902738863

Libkuman, T. M., Stabler, C. L., \& Otani, H. (2004). Arousal, valence, and memory for detail. Memory, 12, 237-247. doi:10.1080/ 09658210244000630

Loftus, E. F., Loftus, G. R., \& Messo, J. (1987). Some facts about "weapon focus. Law and Human Behavior, 11, 55-62. doi:10.1007/BF01044839

McKenna, F. P., \& Sharma, D. (1995). Intrusive cognitions: An investigation of the emotional Stroop task. Journal of Experimental Psychology: Learning, Memory, and Cognition, 21, 1595-1607. doi:10.1037/0278-7393.21.6.1595

Mickley Steinmetz, K. R. (2011). Understanding emotional memory trade-offs: Considering the effect of trait anxiety and post-traumatic stress disorder. (Doctoral dissertation)

Nummenmaa, L., Hyönä, J., \& Calvo, M. G. (2006). Eye movement assessment of selective attentional capture by emotional pictures. Emotion, 6, 257-268. doi:10.1037/1528-3542.6.2.257

Öhman, A., Flykt, A., \& Esteves, F. (2001). Emotion drives attention: Detecting the snake in the grass. Journal of Experimental Psychology. General, 130, 466-478. doi:10.1037/0096-3445.130. 3.466

Reisberg, D., \& Heuer, F. (2004). Remembering emotional events. In D. Reisberg \& P. Hertel (Eds.), Memory and emotion (pp. 3-41). New York: Oxford University Press.

Riggs, L., McQuiggan, D. A., Farb, N., Anderson, A. K., \& Ryan, J. D. (2011). The role of overt attention in emotion-modulated memory. Emotion, 11, 776-785. doi:10.1037/a0022591 
Safer, M. A., Christianson, S.-Å., Autry, M. W., \& Osterlund, K. (1998). Tunnel memory for traumatic events. Applied Cognitive Psychology, 12, 99-117.

Sarter, M., Givens, B., \& Bruno, J. P. (2001). The cognitive neuroscience of sustained attention: Where top-down meets bottom-up. Brain Research Reviews, 35, 146-160.

Schimmack, U., \& Derryberry, D. (2005). Attentional interference effects of emotional pictures: Threat, negativity, or arousal? Emotion, 5, 55-66. doi:10.1037/1528-3542.5.1.55

Shipley, W. C. (1986). Shipley Institute of Living Scale. Los Angeles: Western Psychological Services.

Spielberger, C. D., Gorsuch, R. L., Lushene, R., Vagg, P. R., \& Jacobs, G. A. (1983). Manual for the State-Trait Anxiety Inventory. Palo Alto: Consulting Psychologists Press.

Spreen, O., \& Benton, A. L. (1977). Neurosensory Center Comprehensive Examination for Aphasia: Manual of instructions (NCCEA) (Revth ed.). Victoria: University of Victoria.
Stroop, J. R. (1935). Studies of interference in serial verbal reactions. Journal of Experimental Psychology, 18, 643-662. doi:10.1037/ 0096-3445.121.1.15

Talmi, D., Anderson, A. K., Riggs, L., Caplan, J. B., \& Moscovitch, M. (2008). Immediate memory consequences of the effect of emotion on attention to pictures. Learning and Memory, 15, 172-182. doi:10.1101/lm.722908

Waring, J. D., \& Kensinger, E. A. (2009). Effects of emotional valence and arousal upon memory trade-offs with aging. Psychology and Aging, 24, 412-422. doi:10.1037/a0015526

Waring, J. D., Payne, J. D., Schacter, D. L., \& Kensinger, E. A. (2010). Impact of individual differences upon emotion-induced memory trade-offs. Cognition and Emotion, 24, 150-167. doi:10.1080/ 02699930802618918

Wechsler, D. (1997). Technical manual for the Wechsler Adult Intelligence and Memory Scale-Third Edition. San Antonio: Psychological Corp. 